\title{
Carnets
}

Revue électronique d'études françaises de l'APEF

Deuxième série - 12 | 2018

Théorie Mimétique et Études Littéraires

\section{Job, Meursault, Clamence}

Les guillemets de l'innocence et de la culpabilité

dans la Théorie Mimétique de René Girard

\section{Charles Ramond}

\section{(2) OpenEdition}

1 Journals

\section{Édition électronique}

URL : http://journals.openedition.org/carnets/2412

DOI : 10.4000/carnets. 2412

ISSN : 1646-7698

Éditeur

APEF

\section{Référence électronique}

Charles Ramond, « Job, Meursault, Clamence », Carnets [En ligne], Deuxième série - 12 | 2018, mis en ligne le 31 janvier 2018, consulté le 30 avril 2019. URL : http://journals.openedition.org/carnets/2412 ; DOI : 10.4000/carnets. 2412

Ce document a été généré automatiquement le 30 avril 2019.

\section{(c) (i) (9)}

Carnets est mis à disposition selon les termes de la licence Creative Commons - Atribution - Pas d'utilisation commerciale 4.0 International. 


\title{
Job, Meursault, Clamence
}

\author{
Les guillemets de l'innocence et de la culpabilité \\ dans la Théorie Mimétique de René Girard
}

\section{Charles Ramond}

\section{Introduction}

1 Que ce soit dans sa dimension infantile-égoïste ou dans sa version militante-altruiste, le « sentiment d'injustice », sans doute le plus pur et le plus emblématique des «sentiments moraux", possède une dimension mimétique. Si nous estimons qu'une distribution quelconque (de biens, d'argent, d'honneurs, de reconnaissance) a été faite de façon inégale, nous dirons en effet selon les cas « moi aussi », ou « elle / lui / eux aussi ». Or ces formules («moi aussi », « eux aussi ») sont les formules mêmes de la demande, du désir ou de l'exigence mimétique. Par ailleurs le sentiment d'injustice, sous sa forme altruiste comme sous sa forme égoïste, enveloppe presque toujours, par nature, une certaine violence. Les sentiments moraux (que l'on pense au «respect», au «mépris", à la « honte », à la "pudeur ", à la " colère » ou à « l'indignation », qui est le sentiment moral directement lié au "sentiment d'injustice», et sur lequel je concentrerai ici mes analyses), les sentiments moraux, donc, tirent en effet leur force de leur dimension autoattestative (ils témoignent pour eux-mêmes). Comme la honte par exemple, l'indignation est un sentiment extrêmement vif, violent, impossible à maîtriser, incontestable. En outre, nos sociétés valorisent le plus souvent les sentiments moraux, et tout particulièrement le «sentiment d'injustice » et «l'indignation». De nombreux mythes illustrent dans notre histoire le surgissement de la "légitimité », du sentiment du « juste » et du «bon », face à l'aspect ressenti comme inacceptable de certaines formes de la «légalité »: Antigone, Jeanne d'Arc, le Général De Gaulle, Rosa Parks, et encore récemment les «Indignés » en Espagne (reprenant le titre du célèbre essai de Stéphane Hessel Indignez-vous!), sont ainsi des figures fondatrices, le plus souvent des modèles pour nos sociétés, dressées avec conviction et «indignation » contre certains états des choses -le plus souvent contre des dominations sociales, économiques, politiques ou militaires ressenties comme «injustes». La mythologie américaine montre souvent 
l'affrontement de la « loi » et de la « justice », sous la forme de l'affrontement du « juge » et $d u$ « justicier». Se présentant comme supérieurs à toute convention, les sentiments moraux poussent à sortir de la discussion pour entrer dans l'affrontement, quelle que soit par ailleurs la valeur ou la noblesse de la cause qu'ils prétendent soutenir. Dans tous les cas le « sentiment d'injustice » et « l'indignation » sont vécus comme l'autorisation ou la légitimation d'actions ou de réactions violentes, indépendamment des lois en vigueur, et le plus souvent contre ces lois-mêmes.

2 La Théorie Mimétique ${ }^{1}$ que nous devons à René Girard nous a appris à reconnaitre cette liaison du mimétisme, de la certitude absolue et de la violence. Elle constitue donc un cadre interprétatif et critique particulièrement approprié pour l'étude de ce «sentiment d'injustice» qui nous envahit devant les «injustices» de toute nature. La Théorie Mimétique a pour fonction principale d'atténuer la violence de ce sentiment en nous faisant rétablir les guillemets autour du mot « coupable ». En somme, le Christ serait venu apporter les guillemets au monde... Le discours de la persécution est en effet sans guillemets : il déclare coupable, réellement coupable, coupable-sans-guillemets, celui que l'on veut persécuter. La Théorie Mimétique consiste au contraire à souligner qu'il s'agit d'une accusation, et non pas d'une vérité; pour elle, le persécuté est "accusé", "déclaré » coupable, c'est-à-dire « coupable »-avec-des-guillemets. Les guillemets changent tout : car dès qu'une certitude se reconnaît elle-même comme une accusation, elle perd une très grande partie de sa force, et ne peut plus déboucher directement sur la violence et la persécution. Je souhaiterais, dans les pages qui suivent, développer cette dimension citationnelle de la Théorie Mimétique en mettant en continuité les analyses girardiennes du Livre de Job, et des deux romans de Camus L'Étranger et La Chute, trois textes littéraires entièrement centrés autour des notions de "justice ", de "culpabilité " et d'« innocence». Cela devrait nous conduire à poser une question à la Théorie Mimétique : car si nous devons mettre des guillemets au mot «coupable», ne devrionsnous pas aussi en mettre au mot « innocent »?

\section{Les Justes et les persécuteurs : Job}

3 La Théorie Mimétique, dont le but est de contribuer à libérer les hommes de l'esclavage et de l'enfer du désir mimétique, se réfère à l'enseignement du Christ, qui récuse explicitement l'indignation liée au sentiment d'injustice. La démarche constante du Christ est en effet de rebuter en chacun de nous l'exigence de "justice», si l'on entend par là la proportionnalité des actions et de leurs récompenses, ou l'égale rétribution des travaux et des mérites. C'est le sens de la parabole célèbre entre toutes de "L'ouvrier de la onzième heure $»^{2}$, qui décrit sans ambiguïté ce que nous appellerions une "injustice ». Elle ne donne pas «à travail égal salaire égal ». Les ouvriers ont reçu le même salaire ( "un denier ») pour des horaires de travail inégaux ; ils « murmurent»; ils se plaignent de ce que les derniers embauchés n'ont pas été traités «à l'égal » d'eux-mêmes. Ils sont conscients de l'injustice qui leur est faite, et en souffrent. Or le Christ ne montre aucune compréhension à leur égard, et repousse assez rudement leurs demandes de traitement égal. Certaines des paraboles les plus célèbres du Nouveau Testament vont dans le même sens. Qu'il s'agisse de "Marthe et Marie », du « Fils prodigue », ou du " Figuier desséché », la leçon est la même. L'amour n'est pas une estime. Il n'est pas une juste rétribution. Jésus dit à Marie, restée assise pour l'écouter pendant que sa sœur Marthe s'occupe à organiser la maison pour le recevoir, qu'elle a « choisi la meilleure part ». Le père tue le veau gras 
pour le retour du fils cadet qui est parti et a dépensé tout son héritage, alors qu'il ne fait même plus attention à son fils aîné qui est resté et a aidé aux champs : comportement injuste, et vécu comme tel, avec colère, par le fils aîné3. Un figuier se voit reprocher de ne pas porter de figues, bien que ce ne soit pas la saison. Cette précision est apportée par Marc $(11,13)$. Elle est particulièrement remarquable, car il y a peu d'indications temporelles dans les évangiles. L'injustice du geste du Christ en est soulignée : comment reprocher à un arbre de ne pas porter de fruits quand «ce n'est pas la saison »? Le figuier ne méritait en rien un tel châtiment, qui apparaît donc comme le parangon, délibérément construit et exhibé, d'un châtiment injuste. Or c'est cela (loi d'amour, loi d'injustice) que le Christ est venu apporter, de façon spectaculaire. Le Christ maudit en effet le figuier, et le dessèche du même geste ${ }^{4}$. Rien de plus injuste. Toute la loi nouvelle s'oppose à l'ancienne loi du talion, du « œil pour œil, dent pour dent », donc à toute loi de proportionnalité, mais également à toute conception de reconnaissance des mérites en termes de «juste mesure ", comme on le voit dans Matthieu 25, 29-30, en conclusion de la "parabole des talents ${ }^{5}$. Dans tout cela, on perçoit le fondement d'une vision du monde dans laquelle l'injustice ne peut pas avoir beaucoup de place, parce que la revendication égalitaire y est clairement condamnée.

4 La Théorie Mimétique, appuyée sur la Révélation christique, soutient que le processus d'égalisation mimétique engendre bien plus la guerre que la paix. Mais elle permet d'aller encore plus loin encore dans la critique du « sentiment d'injustice » et de « l'indignation » qui lui est généralement attachée. Il y a "injustice» en effet lorsqu'un innocent est condamné, ou lorsqu'un coupable n'est pas condamné. Or, l'analyse que donne Girard de l'histoire de Job dans La Route Antique des Hommes Pervers conduit à jeter de sérieux doute sur les notions de "culpabilité » et « d'innocence », et, à partir de là, sur la notion même d'injustice-comme je me propose de le montrer maintenant.

5 L'histoire de Job ressemble à la mise en scène d'une injustice maximale. Job était puissant, heureux, et d'un seul coup, sans raison bien claire, le voilà ruiné, rejeté par tous les siens, malade, souffrant. Il a tous les traits d'un innocent injustement condamné par son Dieu. Mais, de façon frappante, Girard ne propose pas de cette histoire une lecture en termes "d'injustice», comme s'il existait une "justice» ordinaire qui, malheureusement, n'aurait pas été respectée dans le cas de Job. Girard voit dans l'histoire de Job un texte de persécution, et dans Job lui-même une figure de bouc émissaire royal persécuté par sa propre communauté. Job était un personnage puissant, et d'un seul coup le voici menacé de mort, entièrement déchu. Rien ne nous dit que Job est un «innocent », au sens où il n'aurait jamais commis aucun méfait, aucun abus de pouvoir, aucune action propre à indigner une foule. Tout au contraire (le texte le laisse entrevoir et Girard le souligne), Job était à ce point puissant qu'il a dû, pendant son règne, commettre bon nombre d'actions répréhensibles à l'égard de ses concitoyens ou de ses sujets. Mais l'essentiel est que, quelles que soient les fautes que Job a pu commettre, ceux qui le condamnent aujourd'hui, et qui s'apprêtent sans doute à le mettre à mort, ou du moins à l'exclure, à l'exiler définitivement, se considèrent et se désignent eux-mêmes comme des « justes ». La petite communauté est maintenant divisée en deux : l'ancien roi, Job, que l'on appelle maintenant "tyran», est considéré comme un "méchant», tandis que ceux qui s'en prennent à lui se déclarent «justes»: "Au spectacle de la chute <des tyrans, des méchants», les justes se réjouissent, et l'homme intègre se moque d'eux ». (Job, 22, 15-20 ; cité in Girard, $1985: 21$ ). 
6 Les "justes » se réjouissent de la chute des "méchants ", et se "moquent d'eux ». Ces hommes sont certainement convaincus eux-mêmes d'être «justes » ou "intègres ». Et pourtant, cela ne fait aucun doute pour Girard, il s'agit d'autant de mensonges. Ces genslà ne sont pas des « justes » mais des « persécuteurs » qui s'ignorent. Le sentiment d'agir «justement » caractérise généralement, en effet, l'absence de doute des persécuteurs. Cette certitude intime et profonde de défendre une cause "juste » pourrait même être considérée par chacun comme un signal d'alerte indiquant l'entrée dans la violence de la persécution. Savoir reconnaître dans la persécution la vérité de la justice, telle serait peut-être la difficile réforme à laquelle nous invite la Théorie Mimétique. Comment saisje que je suis un persécuteur? Je me prends pour un juste... Et qu'est-ce que la certitude? L'entrée dans la persécution...

7 Les persécuteurs (les justiciers) vivent dans un monde où règne « l'injustice » (sous toutes les formes des distributions inégales : pauvreté, oppression, exploitation, corruption, etc.) qui les « indigne » et engendre de leur part des luttes violentes mais « justes ». Comme dit Girard :

«Le narrateur [dans le livre de Job] se présente comme un Juste, un fidèle du vrai dieu, longtemps découragé par l'apparente inertie de la Justice divine. Il fait explicitement état de l'envie que lui inspirait la carrière trop brillante de ceux qu'il présente, bien sûr, comme des impies. Heureusement, le dieu s'est enfin décidé à intervenir » (1985: 68). [Souligné CR]

8 Les majuscules à "Juste " et à "Justice divine » sont de Girard, non pas au sens où il reprendrait à son compte des dénominations valorisantes ("Juste », "Justice divine »), mais dans la mesure où ces majuscules sont comme des guillemets déguisés, des citations ironiques: le narrateur se présente lui-même comme "un Juste ». La majuscule dit la grande estime de soi qu'a le narrateur, sa certitude d'être dans le juste, d'être " un Juste » avec un grand «J ». Pour Girard, bien sûr, le narrateur n'est rien de tout cela : c'est un persécuteur et un menteur...

9 Et lorsque Job s'avise de qualifier sa propre situation « d'injuste », de dire qu'il est, lui, « le juste» et que les autres sont, eux, les «méchants », et que Dieu est «injuste avec lui », d'un seul coup il devient exactement identique à ses persécuteurs. Pour Girard, il est tout aussi inexact et illusoire de considérer Job comme un « Juste » qui serait injustement persécuté par des «méchants", qu'il était illusoire de le considérer auparavant comme un «méchant» justement persécuté par des «justes». Renverser les rôles des persécuteurs et des persécutés n'introduit aucune « justice » dans le système :

«On pense d'abord, sans réfléchir, que ce Dieu, dans sa toute-puissance, userait de ses pouvoirs divins pour empêcher la violence et l'injustice. (...) Ce Dieu des victimes ferait droit, en somme, aux justes revendications de Job. Au lieu de mobiliser ses armées contre le bouc émissaire, il se mobiliserait contre les persécuteurs ». (1985 : 166). [Souligné $C R$ dans tous les cas]

10 Finalement, dans cette histoire de persécution, la justice en tant que telle s'avère insaisissable. Chacun essaie de persécuter l'autre au nom de la justice, ce qui permet à Girard de poser, dans les dernières pages du livre, une question qui résume l'incertitude jetée sur cette question par la Théorie Mimétique - «Dans le cas de Job, qui peut dire où se trouve la justice?»-(1985: 167), jusqu'à une déconstruction presque complète de la notion de justice. 


\section{Justice, vengeance, coupables et victimes}

11 Pour la Théorie Mimétique en effet, il n'y a pas de différence de nature entre la « justice » et la "vengeance ", ni par conséquent entre le système judiciaire moderne et le système sacrificiel archaïque. Girard s'explique sur ce point aux pages 29 et suivantes de $L a$ violence et le sacré :

Le système judiciaire écarte la menace de la vengeance. Il ne supprime pas la vengeance: il la limite effectivement à une représaille unique dont l'exercice est confié à une autorité souveraine et spécialisée dans son domaine. (...) Il n'y a, dans le système pénal, aucun principe de justice qui diffère réellement du principe de vengeance. Le même principe est à l'œuvre dans les deux cas, celui de la réciprocité violente, de la rétribution. Ou bien ce principe est juste et la justice est déjà présente dans la vengeance, ou bien il n'y a de justice nulle part ». (1972: 29-30). [Souligné CR dans tous les cas]

12 De ce fait, le système judiciaire se distingue du système sacrificiel seulement par son efficacité supérieure dans le maintien de l'ordre public. Girard aurait pu reprendre entièrement à son compte la fameuse Pensée de Pascal : « Ne pouvant faire que ce qui est juste fût fort, on a fait que ce qui est fort fût juste». Du point de vue de la Théorie Mimétique, la «justice» devient ainsi un autre nom du «maintien de l'ordre», ou du rapport des forces en un lieu et un moment donnés d'une société. Les notions mêmes de " justice » et "d'injustice ", et les sentiments qui sont liés à ces notions, sont considérés par Girard comme de simples illusions: "Derrière la différence à la fois pratique et mythique, il faut affirmer la non-différence, l'identité positive de la vengeance, du sacrifice et de la pénalité judiciaire ». (1972: 42).

13 La déconstruction de la notion de justice s'étend en outre aux notions de " culpabilité » et « d'innocence ». La thèse de Girard va être en effet que les « victimes » seules sont réelles, tandis que les « coupables » comme les « innocents » relèvent de l'imaginaire.

14 Comment distinguer «victime» et «coupable»? Du point de vue de la Théorie Mimétique c'est moins facile qu'on ne pourrait le croire. La Théorie Mimétique nous conduit en effet à faire porter un doute méthodologique sur la notion (et le terme même) de «culpabilité » : si la « justice» ne se distingue pas d'une "vengeance», si sa fonction est non pas de dire le «juste » ou "l'injuste », mais de rétablir l'ordre et la paix dans la communauté, alors nous pouvons toujours soupçonner qu'un « coupable » est en réalité une « victime » qui a été « accusée » de certains crimes tout particulièrement propices à la faire passer du statut d'accusée à celui de victime. Nous devrions donc toujours mettre des guillemets autour du mot « coupable » : car « coupable » a toujours pour référent un discours accusatoire. «Coupable » est un terme intrinsèquement citationnel, qui signifie "déclaré coupable». "Coupable sans guillemets» (difficile à dire, n'est-ce pas? Quel geste faire ici avec les mains et les doigts ?...) est sans doute le chaînon manquant entre «accusé » et «victime ». Enlever les guillemets à «coupable", tout comme ôter ses protections à un accusé, c'est produire le basculement dans la violence.

$15 \mathrm{Au}$ fond, contre quoi ou contre qui s'indigne-t-on ? N'est-ce pas nécessairement contre un «coupable sans guillemets»? On ne pourrait pas s'indigner, en effet, ou se révolter, contre quelqu'un (Ministre, homme d'affaires, Président, Directeur de banque ou d'institution internationale) que l'on " présumerait innocent ». L'indignation a besoin de certitude, le doute la fait disparaître. Et donc, lorsque nous nous « indignons » contre un système économique (que ce soit le socialisme ou le capitalisme) ou contre un 
gouvernement (parce qu'il y a du chômage, parce que des gens dorment dans les rues), c'est bien parce que nous les tenons pour des "coupables sans guillemets». Mais comment pouvons-nous savoir qu'un système économique ou un gouvernement sont « coupables sans guillemets » de l'ordre des choses ? Où a été fait leur procès? Par qui a-til été tranché? Un tel procès serait sans doute impossible, pour des raisons à la fois matérielles (on ne peut pas faire comparaitre «le capitalisme» devant un tribunal), politiques (aucun gouvernement n'accepterait d'être jugé dans un tribunal parce qu'il n'aurait pas réussi à faire baisser le chômage), et logiques (pour « instruire le procès » du " capitalisme » il faudrait définir d'abord le « capitalisme », et entrer de ce fait dans une régression à l'infini - car quel tribunal pourrait trancher de la validité d'une telle définition ?). Bien loin donc de pouvoir condamner le capitalisme comme « coupable sans guillemets ", nous ne pouvons même pas espérer, faute de tribunal compétent, le voir qualifié un jour de "coupable avec guillemets", c'est-à-dire de "déclaré coupable». Notre indignation à son égard ne peut et ne pourra donc que manquer d'aliments juridiques.

16 Ce discours sera sans doute un peu difficile à entendre. Nous avons tellement besoin de coupables... Nous avons tellement envie de les désarmer, de leur arracher l'armure des guillemets... Nous aimerions tellement avoir accès à la réalité (toute nue ?) et non pas à des discours... Nous sommes tellement avides d'unanimité et de certitude sur ce genre de sujets... Mais justement la Théorie Mimétique ne cesse de nous mettre en garde contre ce genre de désirs de culpabilités objectives, de certitudes, de châtiments, désirs de violence toujours... Le coupable sans la protection des guillemets sera très vite une victime.

On s'insurgera peut-être, d'un point de vue logique, contre de telles conclusions, et l'on pensera sans doute absurde de remettre en question le fait que le système judiciaire produit des coupables réels, des « coupables sans guillemets ». L'obsession du système judiciaire n'est-elle pas, justement, de découvrir les "vrais coupables», d'éviter toute " erreur judiciaire»? N'avons-nous pas les «experts», la "police scientifique», les preuves, les empreintes digitales, bref tous les moyens de trouver les « vrais coupables»? Le travail de la justice ne consiste-t-il pas, précisément, dans la "décitation», ou suppression des guillemets autour du mot « coupable »?

18 Pourtant, même cela pourrait être discuté. D'abord parce qu'une action n'admet pas une description unique. Ai-je «appuyé sur la gâchette » ou «tué l'âne de mon voisin »? Ce sont deux descriptions concurrentes, et il y en aurait une infinité d'autres possibles. Et quand bien même on aurait réussi à s'accorder sur la description d'une action, il resterait à savoir en quelle mesure on la considère comme un crime qui doit ou non être puni. C'est très variable d'un lieu à un autre ou d'un temps à un autre, comme on peut le voir en ce qui concerne l'adultère, l'homosexualité, la prostitution, le viol, les mauvais traitements aux animaux, le fait de fumer, etc. La loi vous déclare " coupable » à un certain moment pour une certaine action, et « innocent » à un autre moment pour la même action. Donc on peut toujours garder la sensation d'un arbitraire social : ce qui était cherché, ce n'était pas tant le vrai coupable, au sens de celui qui aurait vraiment, objectivement, fait quelque chose de mal, mais la victime qu'on pouvait condamner de la façon la plus efficace pour rétablir l'ordre social avec le moins de dépense d'énergie possible. Des "coupables » toujours entre guillemets, mais des victimes bien réelles... 


\section{L'Étranger et La Chute : peut-on être « réellement coupable »?}

19 La Théorie Mimétique, pour nous délivrer de la violence unanime du mimétisme, propose ainsi une vision sceptique et relativiste des notions de justice, d'injustice et de culpabilité. Derrière le "coupable ", elle nous apprend à reconnaître une victime, derrière les «justes », des persécuteurs, et derrière la «justice», une vengeance institutionnelle ayant pour finalité de maintenir l'ordre dans une communauté. Par-là se révèle la parenté profonde entre la Théorie Mimétique et la Déconstruction derridienne. À la p. 69 de La Violence et le sacré, Girard met ainsi sur le même plan, contrairement à nos attentes les plus constantes, les termes " criminel », « justicier », et « sacrificateur ». Nous aimerions en effet qu'un «juge» soit tout autre chose qu'un «justicier», un «criminel», un "vengeur», ou un "sacrificateur». Mais Girard estime que ces personnages sont en réalité très proches les uns des autres. De même nous aimerions qu'un « philosophe » soit tout autre chose qu'un « sophiste ", qu'un « loup » soit tout autre chose qu'un « chien", et qu'un "poison » soit tout autre chose qu'un « remède » (bien qu'ils portent en grec tous deux le même nom de pharmakon). La Théorie Mimétique insiste au contraire sur l'«indécidabilité » des figures conceptuelles de la justice comme de l'anthropologie générale, puisque le "bouc émissaire » y est à la fois un " poison » aux yeux de la société et le "remède » à ce poison. La Théorie Mimétique nous plonge même directement dans la "citationnalité ", puisque pour elle "coupable " est toujours déjà une "citation ", renvoie toujours à un autre discours, et jamais à une réalité.

Girard sait bien ce qu'il doit à Derrida, et à quel point la Théorie Mimétique est souvent proche de la Déconstruction. Pour autant, il a toujours perçu assez étrangement dans la philosophie de Derrida un relativisme, un scepticisme, une indifférence à la vérité, un éloignement du « réel », auquel il a toujours voulu opposer une doctrine « réaliste ${ }^{6}$. On doit donc s'attendre à trouver chez Girard, au milieu des thèses sceptiques et relativistes (sur la justice, l'injustice, et la culpabilité) que je viens d'exposer, de soudaines réactions allant dans le sens contraire, et dans lesquelles se fait voir ce souci profond chez Girard de ne pas quitter la « réalité ». Autrement dit, on doit s'attendre à voir Girard, comme Job lui-même (dont il se sentait parfois, sans doute, assez proche en tant que victime d'une sorte d'expulsion théorique presque généralisée, du moins dans son propre pays...), tenir parfois le discours de ses adversaires, de ceux qui lui sont le plus opposés théoriquement. Il est sans doute impossible en effet de ne pas être contaminé mimétiquement, de temps à autre, par les discours qui circulent sans cesse autour de vous.

21 Ce double discours (ou, si l'on préfère, ce recul par rapport aux avancées de la Théorie Mimétique) est particulièrement visible dans la très remarquable interprétation que propose Girard du roman de Camus L'Étranger (« Pour un nouveau procès de L'Étranger ", paru en anglais en 1964, puis en français en 1968).

Girard propose une lecture extrêmement critique de L'Étranger, en s'appuyant sur La Chute , dans lequel il voit une auto-critique de Camus, et son authentique chef-d'œuvre. L'Étranger correspondrait, dans l'écriture et dans la vie de Camus, à une période romantique et infantile. L'auteur, par le biais de son héros Meursault, se serait engagé dans un geste contradictoire : clamer son indifférence à l'égard de la société en général, et en même temps faire un geste répréhensible qui fasse que la société remarque cette indifférence. De ce fait, Camus aurait essayé de construire dans L'Étranger la figure 
impossible d'un «meurtrier innocent ». Girard oppose à cette posture d'« indignation » et d'« authenticité », propre au " romantisme » juvénile de l'auteur, d'une part une défense de la justice institutionnelle, et d'autre part le fait que, dans La Chute, Camus renie tout ce qu'il soutenait dans L'Étranger, par une sorte de conversion très semblable à celles que Girard a étudiées dans Mensonge Romantique et Vérité Romanesque, à la fin des «grands romans", où le héros renonce à la fascination haineuse qu'il ressentait envers ses semblables, et se délivre du mimétisme en acceptant d'être semblable à eux.

Donc, d'un côté la lecture de L'Étranger par Girard est conforme dans ses grandes lignes à la Théorie Mimétique. Il s'agit d'y décrire un auteur essayant en vain de se délivrer de l'esclavage du mimétisme, et de mettre en valeur les postures "d'indignation " et « d'authenticité » par lesquelles il cherche à se prétendre « innocent » tout en rendant la société (et surtout ses juges) «coupables» du sort qu'il subit. Mais d'un autre côté, puisque Girard ne veut pas accepter la fiction construite par Camus-Meursault d'un "meurtrier innocent », il est contraint, dans son interprétation, de considérer Meursault comme réellement coupable, comme « coupable sans guillemets » - et là, pour le coup, en contradiction avec ce que nous avons vu être le cœur de la Théorie Mimétique.

Bien plus, le fait que Girard veuille à tout prix que Meursault soit « réellement coupable » de son meurtre l'empêche, de façon tout à fait incroyable, de voir le parallèle, pourtant patent, entre Meursault et Job. En général, Girard repère facilement ce genre de schème : le « coupable » est en fait une " victime »; «l'accusé » est en fait « innocent ». Il en fait même le schème fondamental de son anthropologie. Comment donc a-t-il pu ne pas le voir à propos du roman de Camus? Supposons qu'il s'agisse d'ÆEdipe : Girard dirait-il qu'CEdipe est condamné à bon droit, et qu'il a tort de se plaindre de ses juges, parce qu'il a «réellement commis » deux crimes, tuer son père et épouser sa mère ? Certainement pas: la Théorie Mimétique nous a appris au contraire à voir là un discours-type de persécution, et Girard a beaucoup insisté, dans La violence et le sacré, sur le fait que CEdipe n'est pas bien certain d'avoir commis les crimes dont on l'accuse.

Mutatis mutandis, L'Étranger de Camus aurait dû être l'objet de la même interprétation. Quel est aujourd'hui en effet le pire des crimes dont on puisse accuser quelqu'un? Celui qui vous conduit tout droit au lynchage ? Est-ce tuer son père et désirer sa mère? Bien sûr que non: ces actes sont devenus même presque obligatoire pour nous autres freudiens. Celui qui ne voudrait pas tuer son père et ne désirerait pas sa mère nous semblerait étrange... D'ailleurs, significativement, L'Étranger s'ouvre sur l'indifférence du narrateur à l'égard de sa mère, indifférence qui lui sera reprochée dans le roman, et qui sera l'une des raisons qui le feront condamner à mort. Si au moins Meursault avait voulu coucher avec sa mère, il aurait sans doute eu l'indulgence du jury... De nos jours, l'accusation suprême n'est plus l'œedipe, mais le crime raciste. En cela, le roman de Camus touche juste. Qui est accusé d'avoir commis un crime raciste (comme dit sans cesse Camus, repris sans guillemets par Girard, de "tuer un arabe»), subira le lynchage contemporain, c'est-à-dire le lynchage médiatique, et sera en outre très lourdement condamné. Le crime raciste est aujourd'hui l'accusation persécutrice type, le nouveau sacré. Le meurtrier raciste est le pire des hommes, exactement comme đEdipe à Thèbes alors que dans le monde antique la notion de "crime raciste", bien loin d'être condamnable, n'existait tout simplement pas. Donc Girard aurait pu repérer dans l'Étranger l'accusation persécutrice typique de nos sociétés démocratiques et ouvertes. D'autant plus, Camus y insiste, et Girard est agacé de la réussite technique du roman justement sur ce plan, d'autant plus, dis-je, que Meursault n'est pas bien certain d'avoir 
commis un crime. Tout le roman est en effet construit pour que ce meurtre soit en quelque sorte détaché de son auteur: il est commis dans une sorte d'hallucination, d'extase mystique, affectif, naturaliste, où Meursault, de toute évidence, est hors de lui-même, ne sait pas ce qu'il fait, n'est pas vraiment présent à cette scène, comme s'il était dans un rêve. ${ }^{7}$

Tous les éléments étaient donc réunis pour déceler dans L'Étranger de Camus un roman du bouc émissaire contemporain : quelqu'un qui s'estime innocent alors que toute la société l'estime coupable, et le condamne à mort. D'ailleurs Girard, presque instinctivement, avait repris le vocabulaire de la "persécution » dans un passage du début de l'article: « En fin de compte, Meursault est un petit bureaucrate sans ambition et en tant que tel rien ne le destine à être persécuté ». (1976:149). [Souligné CR]

Quelque lignes plus loin, Girard écrivait que Camus voulait faire de son héros un " martyr », et que pour cela il fallait qu'il lui fasse faire un acte "répréhensible », mais dont il soit cependant « innocent »; et à la fin de l'article, Girard comparait Meursault à CEdipe, " autre héros de la littérature de procès ", mais comme en passant et comme s'il n'avait pas encore remarqué, à l'époque où il écrivait cet article, que đEdipe pouvait très bien ne pas avoir commis les crimes dont on l'accusait.

Malgré ces quelques infléchissements, Girard reprend pourtant dans son article, pour l'essentiel, le discours des persécuteurs. Meursault «est coupable, parfaitement coupable "; il a "réellement commis un crime ", c'est l'essentiel, c'est pour cela qu'il est condamné à mort. Sa plainte contre les juges n'est pas celle d'un innocent injustement accusé, comme il le prétend, c'est la plainte infantile d'un enfant boudeur et qui veut se faire remarquer, comme l'écrivain Camus voulait se faire remarquer du public. Meursault est « coupable sans guillemets ». On a presque l'impression que Girard pourrait ici tenir le rôle des fameux "amis» de Job, qui veulent absolument lui faire endosser la responsabilité du châtiment qui lui arrive... Girard est tellement énervé (indigné ?) contre la prétention de Camus à avoir présenté un héros qui soit à la fois «meurtrier » et "innocent", qu'il va jusqu'à souligner en italiques 5 lignes entières de son texte (fait suffisamment rare pour être noté) pour bien insister sur la culpabilité réelle de Meursault et sur l'incohérence générale du roman :

On nous conduit insensiblement à l'incroyable conclusion que le héros est condamné à mort non pour le crime dont il est accusé et dont il est réellement coupable, mais à cause de son innocence que ce crime n'a pas entachée, et qui doit rester visible aux yeux de tous comme si elle était l'attribut d'une divinité (1976: 151; Italiques de Girard, Souligné $\mathrm{CR})$.

Mais Girard n'est-il pas le premier à savoir et à dire que celui que tous accusent et veulent condamner à mort n'est sans doute pas aussi « coupable » que le dit la foule, et peut-être pas tellement plus coupable que chacun de ceux qui l'accusent? Que c'est là l'histoire même de l'humanité?

\section{Conclusion : l'introuvable innocence}

Toutes ces analyses nous conduisent, pour conclure, à mettre en lumière et à expliquer une difficulté assez inattendue de la Théorie Mimétique, concernant la notion "d'innocence ». On dit souvent que la Théorie Mimétique nous apprend à reconnaître "l'innocence » des boucs-émissaires, des persécutés. Tel serait le message porté au plus haut point par le Christ, et avant lui par toutes les Figurae Christi (Job, la «bonne mère » 
du jugement de Salomon, et tous ceux qui ont essayé de briser l'unanimité violente du mimétisme persécuteur). Pourtant, à bien y réfléchir, la Théorie Mimétique ne peut pas dire exactement cela. Car, selon cette théorie, il n'existe pas plus d'« innocents sans guillemets » que de " coupables sans guillemets ». La Théorie Mimétique nous apprend en effet à renoncer à un monde partagé entre des individus « réellement coupables » d'un côté et des individus "réellement innocents» de l'autre, et à comprendre (comme l'illustre la parabole de la "femme adultère») que nous sommes tous identiquement «coupables" et "innocents» à des degrés divers, mais jamais totalement l'un ni totalement l'autre, et que l'humanité ne se divise pas, sauf dans les mythes, en coupables et innocents, pas plus qu'entre Justes et méchants. Par conséquent, lorsqu'il nous arrive de trouver, dans le cadre de la Théorie Mimétique, des déclarations sur l'innocence réelle ou la culpabilité réelle de certains individus, nous savons que nous sommes momentanément en train de lire le contraire de ce que soutient la Théorie Mimétique. Pour parvenir sinon à supprimer entièrement, du moins à réduire les sources et les occasions de la violence mimétique, la Théorie Mimétique ne devrait pas hésiter à aller jusqu'au bout de son scepticisme, de son relativisme, et maintenir sa distance au « réalisme ». Comme le montrent les analyses girardiennes du livre de Job et des romans de Camus, jusque dans les difficultés qu'elles rencontrent, la Théorie Mimétique permet en effet de relativiser, déconstruire, ou citationnaliser les notions de "justice», " injustice », « culpabilité », « innocence », et par conséquent tous les sentiments moraux qui leur sont liés et les certitudes violentes qui en découlent.

\section{BIBLIOGRAPHIE}

Ancien et Nouveau Testament, Edition Bible de Jérusalem (1980).

Bove, Laurent (2014). Albert Camus. De la transfiguration. Paris : Publications de la Sorbonne.

CAMus, Albert (1942). L'Étranger. Paris : Gallimard.

CAMUS, Albert (1956). La Chute. Paris : Gallimard.

DERRIDA, Jacques (1968). « La Pharmacie de Platon », Tel Quel 32 et 33.

HESSEL, Stéphane (2010). Indignez-vous !, Montpellier : Indigènes Éditions.

GIRARD, René (1963). Mensonge romantique et vérité romanesque. Paris : Grasset.

GIRARD, René (1972). La Violence et le Sacré. Paris : Grasset.

GIRARD, René (1976). « Pour un nouveau procès de L'Étranger », in Critiques dans un souterrain.

Paris : Livre de Poche.

GIRARD, René (1978). Des Choses cachées depuis la fondation du monde - Recherches avec Jean-Michel Oughourlian et Guy Lefort. Paris : Grasset.

GIRARD, René (1985). La Route antique des hommes pervers. Paris : Livre de Poche.

GIRARD, René (2001). Celui par qui le scandale arrive. Paris : Desclée de Brouwer. 
GIRARD, René (2002). La Voix méconnue du réel. Paris : Grasset.

GIRARD, René (2007). Achever Clausewitz. Paris : Carnets Nord.

PASCAL, Blaise [1662] (2010), Pensées, édition de Philippe Sellier, Paris : Classiques Garnier.

\section{NOTES}

1. Nous écrivons "Théorie Mimétique" avec des majuscules, en considérant ainsi cette expression comme un nom propre désignant la théorie développée d'abord dans les œuvres de René Girard. Pour les mêmes raisons, nous écrirons un peu plus loin « Déconstruction » avec une majuscule.

2. «Car le royaume des cieux est semblable à un maître de maison qui sortit dès le matin, afin de louer des ouvriers pour sa vigne. Il convint avec eux d'un denier par jour, et il les envoya à sa vigne. Il sortit vers la troisième heure, et il en vit d'autres qui étaient sur la place sans rien faire. Il leur dit : Allez aussi à ma vigne, et je vous donnerai ce qui sera raisonnable. Et ils y allèrent. Il sortit de nouveau vers la sixième heure et vers la neuvième, et il fit de même. Étant sorti vers la onzième heure, il en trouva d'autres qui étaient sur la place, et il leur dit : Pourquoi vous tenezvous ici toute la journée sans rien faire ? Ils lui répondirent: C'est que personne ne nous a loués. Allez aussi à ma vigne, leur dit-il. Quand le soir fut venu, le maître de la vigne dit à son intendant : Appelle les ouvriers, et paie-leur le salaire, en allant des derniers aux premiers. Ceux de la onzième heure vinrent, et reçurent chacun un denier. Les premiers vinrent ensuite, croyant recevoir davantage ; mais ils reçurent aussi chacun un denier. En le recevant, ils murmurèrent contre le maître de la maison, et dirent: Ces derniers n'ont travaillé qu'une heure, et tu les traites à l'égal de nous, qui avons supporté la fatigue du jour et la chaleur. Il répondit à l'un d'eux : Mon ami, je ne te fais pas tort; n'es-tu pas convenu avec moi d'un denier ? Prends ce qui te revient, et va-t'en. Je veux donner à ce dernier autant qu'à toi. Ne m'est-il pas permis de faire de mon bien ce que je veux? Ou vois-tu de mauvais œil que je sois bon? Ainsi les derniers seront les premiers, et les premiers seront les derniers ». (Matthieu 20, 1-16).

3. Tout le contexte de Luc 5 prépare cette parabole : « Il y aura de la joie dans le ciel, pour un seul pécheur qui se convertit, plus que pour quatre-vingt-dix-neuf justes qui n'ont pas besoin de leur conversion ». C'est une négation explicite de l'égalité arithmétique, et, à travers cela, de tout traitement égalitaire. La parabole de la «brebis égarée» comme celle du «fils prodigue » décrivent ce que nous appellerions sans aucun doute des « injustices». Et c'est cela que le Christ demande d'accepter.

4. C'est la version de Matthieu (21, 18 et suiv.). Dans celle de Luc (13, 6-9), Jésus montre un peu plus de patience.

5. «Car à tout homme qui a, l'on donnera et il aura du surplus; mais à celui qui n'a pas, on enlèvera ce qu'il a. Et ce propre à rien de serviteur, jetez-le dehors, dans les ténèbres : là seront les pleurs et les grincements de dents ». (Matthieu 25, 29-30).

6. La question du « réalisme » est sensible et difficile chez Girard. Il y revient souvent. Dans Le bouc émissaire, il justifie son « réalisme » à propos du texte de Guillaume de Machaut, Jugement du Roy de Navarre: nous devons savoir déceler, dans les "textes de persécution ", des accusations fantasmatiques (il a plu des pierres) et des éléments "réalistes" (les juifs ont été massacrés). Dans son dernier livre Achever Clausewitz, Girard s'est exprimé aussi à l'égard de l'innocence des victimes ou sur la question du réalisme : « Nous avons un rendez-vous avec le réel » (2007: 209). Dans tous les cas, il s'agit d'un «demi-réalisme » ou d'un réalisme "pondéré »: nous devons apprendre à distinguer les persécutions « réelles » et les accusations « imaginaires ». Lévi-Strauss et toute la lecture structuraliste des mythes n'ont pas su voir la « réalité » des persécutions et des violences (voir La Violence et le sacré, chp. 9, et Des Choses cachées, I, chp. 4 et 5. Un des livres de 
Girard s'intitule La voix méconnue du réel, et le premier chapitre de Celui par qui le scandale arrive s'appelle «Contre le relativisme». Voir également C. Ramond, Le vocabulaire de Girard, Article "Vérité romanesque, mensonge romantique, réalisme». Il n'en reste pas moins - telle est la thèse que nous soutenons ici - qu'on ne peut pas soutenir à la fois le caractère imaginaire de la culpabilité et le caractère réel de l'innocence.

7. Pour une lecture spinoziste de l'Étranger, et de cette fusion avec le monde, voir l'ouvrage de Laurent Bove, Albert Camus. De la transfiguration, Paris : Publications de la Sorbonne, 2014.

\section{RÉSUMÉS}

La Théorie Mimétique distingue accusation et culpabilité. đEdipe est «accusé » d'avoir tué son père et commis l'inceste avec sa mère; cela ne signifie pas qu'il est «coupable». Dans les Évangiles, une femme est "accusée » d'adultère; mais en déclarant «que celui qui n'a jamais péché jette la première pierre", le Christ fait comprendre que la femme n'est pas plus «coupable» que ceux qui l'accusent. Toute la théorie mimétique peut donc se résumer à une affaire de guillemets. Lorsque l'on enlève les guillemets au mot "coupable ", lorsqu'on parle donc de "coupables sans guillemets ", on crée des victimes que l'on s'apprête à persécuter. Cet article met en évidence l'importance des guillemets dans les analyses par Girard du Livre de Job, et des deux romans de Camus L'Étranger et La Chute. Ces analyses débouchent sur une question adressée à la Théorie Mimétique : si «coupable» doit être toujours écrit entre guillemets, ne doit-il pas en aller de même pour « innocent »?

Mimetic Theory distinguishes accusation and guilt. Oedipus is "accused " of having killed his father and committed incest with his mother; it does not mean that he is "guilty ". In the Gospels, a woman is « accused » of adultery; but by declaring « that he who has never sin cast the first stone ", Christ makes it clear that this woman is no more " guilty » than those who accuse her. All the Mimetic Theory can then be summarized as a question of quotation marks. When one removes the quotation marks from the word "guilty", when one so speaks of "guilty without quotation marks", one creates victims that one is about to persecute. This article highlights the importance of quotation marks in Girard's analysis of the Book of Job, and the two novels by Camus L'Étranger and La Chute. All of this lead to a question addressed to Mimetic Theory: If " guilty » must always be written in quotation marks, should it not be the same for " innocent»?

\section{INDEX}

Mots-clés : théorie mimétique, guillemets, justice, innocence, culpabilité

Keywords : mimetic theory, quotation marks, justice, innocence, guilt

\section{AUTEUR}

CHARLES RAMOND

Université Paris 8

charles.ramond[at]univ-paris8.fr 